\title{
Reseña sobre La Revolución Google Scholar. Destapando la caja de Pandora académica
}

\author{
Sandra Miguel * \\ * Instituto de Investigaciones en Humanidades y Ciencias Sociales (IdIHCS) (UNLP- \\ CONICET), Facultad de Humanidades y Ciencias de la Educación, Universidad Nacional de \\ La Plata, Argentina | smiguel@fahce.unlp.edu.ar
}

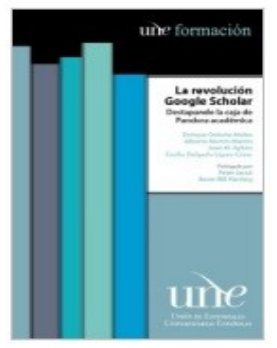

Reseña de:

La Revolución Google Scholar. Destapando la caja de Pandora académica / Enrique Orduña Malea, Alberto Martín-Martín, Juan M. Ayllón y Emilio Delgado López Cózar (2016). Granada: Unión de Editoriales Universitarias Españolas. 266 p.

La amplia cobertura de Google Scholar, el buscador académico que indiza documentos escritos en cualquier lengua, procedentes de cualquier país, bajo cualquier formato y tipología documental, parece rememorar el sueño del Repertorio Bibliográfico Universal de los investigadores belgas Paul Otlet y Henri Lafontaine, en las postrimerías del siglo XIX. En aquel entonces surgía el catálogo bibliográfico basado en el uso de las fichas móviles y en el lenguaje de la Clasificación Decimal Universal, concebido como una nueva y revolucionaria herramienta de acceso al conocimiento en todo el mundo. Luego de muchas décadas, en el presente siglo, una nueva revolución en la recuperación de información viene de la mano de Google Scholar, que está transformando la forma de buscar, encontrar y acceder a la información científica en el mundo entero. Pero además de su perfil revolucionario como motor de búsqueda, la otra cara que hace a Google Scholar cobrar cada vez más notoriedad e importancia entre los miembros de la comunidad académica y estudiosos de la bibliometría radica en su potencial utilidad como fuente de 
datos para la evaluación de la producción e impacto científicos.

Prologado por dos reconocidos estudiosos de Google Scholar, el profesor Peter Jacsó de la Library and Information Science Program, Department of Information and Computer Science, Honolulu, Hawaii, y la profesora Anne-Wil Harzing, de la Middlesex University London; seguido de una revisión omnicomprensiva y minuciosa sobre lo que es a día de hoy Google Scholar y sus derivaciones, los contenidos de la obra se estructuran en dos grandes bloques ( $\mathrm{A}$ y $\mathrm{B}$ ), un apartado de conclusiones y otro conteniendo una profusa cantidad de bibliografía actualizada y pertinente que hace que la obra aporte un verdadero estado de la cuestión sobre el tema.

El bloque A presenta a Google Scholar como el buscador académico universal. En los siete capítulos que abarca realiza una exhaustiva y minuciosa revisión de sus características y servicios. El capítulo 1 traza un recorrido desde su origen y evolución desde 2004 a 2014. Los capítulos 2 y 3 presentan una revisión bibliográfica crítica de los principales hallazgos y discusiones sobre este buscador; en primer lugar, referida a las opiniones y a las percepciones de sus usuarios como herramienta de búsqueda de material académico, y en segundo lugar, de aquellos que lo estudian como objeto para analizar su validez como herramienta para la evaluación de la actividad científica a partir de indicadores bibliométricos. El capítulo 4 realiza un análisis del funcionamiento del motor de búsqueda detallando en forma minuciosa el proceso y los requisitos de indización de los documentos. El capítulo 5 describe las características del buscador desde la perspectiva de su cobertura, tamaño y evolución. El capítulo 6 detalla el proceso de la búsqueda y sus principales prestaciones y servicios. El bloque finaliza con el capítulo 7, en el que los autores realizan una valoración crítica de las fortalezas, las debilidades y los peligros de las dos caras de este buscador.

El bloque B, que comprende tres capítulos, enfoca la atención en la descripción y el análisis de los principales productos derivados del buscador, y desarrollados como herramientas para la evaluación científica con múltiples usos bibliométricos. Así, el capítulo 8 está dedicado a mostrar las características, las bondades y las limitaciones de Google Scholar Metrics, un servicio pensado para medir el impacto de las revistas científicas, que las presenta en un ranking de títulos clasificados en diferentes campos del conocimiento e idiomas y ordenados por el valor del índice $\mathrm{h}$ que reciben las publicaciones. El capítulo 9 describe las características de Google Scholar Citations, creado como un servicio a los autores para generar perfiles bibliográficos. Esta herramienta que ha ganado gran popularidad en la comunidad científica muestra la producción de los investigadores que es recogida por el buscador, y genera una serie de indicadores bibliométricos de impacto basado en el recuento de las citas recibidas por esos documentos en el universo Google Scholar. Además de esos dos productos oficiales creados por el mismo equipo que desarrolla el buscador, el capítulo 10 dedica su atención a otro conjunto de desarrollos independientes que han surgido como herramientas para análisis bibliométricos utilizando la colección de este repertorio como fuente de información: Publish or Perish, Scholarometer, H index Scholar, Journal Scholar Metrics, Publisher Scholar Metrics, Proceedings Scholar Metrics y Scholar Mirrors. Estos productos se complementan ofreciendo diferentes métricas resultado de la aplicación de algoritmos de recuperación de información e indicadores bibliométricos. 
De la lectura atenta del contenido de sendas secciones del libro es posible apreciar las numerosas bondades y ventajas de Google Scholar; pero también de sus problemas y limitaciones, lo que hace que la posición hegemónica alcanzada para la búsqueda de material académico no se haya trasladado, al menos por ahora, a su aceptación como fuente válida y confiable para la evaluación de los investigadores y de los recursos y productos de la actividad científica y tecnológica.

Las conclusiones de la obra, que recogen de manera resumida sus principales ideas, retoman el concepto que muy acertadamente aparece expresado en el título del mismo, porque pese a los numerosos estudios que se han realizado desde su aparición en 2004 hasta la actualidad, la "caja de Pandora académica", aún sigue siendo el lema que representa a Google Scholar. Su apertura y mayor conocimiento solo será posible siguiendo el camino de la investigación que los especialistas en el campo de la Ciencia de la Información y de la Bibliometría ya han comenzado a recorrer, y cuyos frutos, como es el resultado de la magnífica obra que se reseña ayudarán a descubrir.

En un balance general, la obra representa una valiosa contribución al conocimiento de esta nueva y revolucionaria herramienta de búsqueda de información y potencial fuente de evaluación de la ciencia que se realiza desde la Ciencia de la Información a todos los campos del saber científico y tecnológico. 